\title{
Slowing down a "slow" movement disorder: Selegiline's benefit in treating Parkinson disease
}

Steven E. Lo, MD; and Samay Jain, MD

Parkinson disease (PD) is a chronic disorder of the nervous system that is characterized by tremors, slowness of movement, rigidity, and balance problems. There is no single "right way" to treat patients who have early stage PD and mild symptoms. One strategy is to use a medication called selegiline, which has been proven to relieve many symptoms in PD and may help delay the need to add stronger medications. In this issue of Neurology, Dr. Pålhagen and his colleagues ${ }^{1}$ report on a study that evaluated if selegiline slows down the progress of symptoms in patients with PD who need to start the most effective of PD medications, levodopa. More information on PD and levodopa can be found on the next page.

\section{What is selegiline?}

Selegiline is a medication in the monoamine oxidase type $B$ (MAO-B) inhibitor family of drugs. MAO is a protein in cells that can break down dopamine, the main chemical that is deficient in PD. Therefore, by inhibiting MAO, selegiline helps keep dopamine available for brain cells to use. Selegiline also helps keep any replacement dopamine (created from levodopa) from being broken down.

\section{How was the study done?}

Dr. Pålhagen reported on the results of a long study lasting 7 years in which selegiline plus levodopa was compared to placebo (a pill that looks like selegiline but does not contain medication) plus levodopa in a group of patients with PD. The goal of the study was to see what effect early and continued use of selegiline would have in the progression of PD symptoms.
Levodopa was started on all patients in either group (selegiline or placebo) who needed a stronger medication because their PD symptoms had worsened over the years. These patients were then followed regularly for the next several years. At each evaluation, the investigators recorded how severe the patients' PD symptoms were (using a scale called the Unified Parkinson Disease Rating Scale [UPDRS]; see the next page), how much levodopa was needed to treat PD symptoms, any complaints of side effects from the medications, and any complaints of dyskinesias (see next page) or "wearing-off" fluctuations (see next page). After the patients were studied for a total of 7 years, the information between the two groups was compared.

\section{What were the results of the study?}

Twenty-nine of the 72 patients who got selegiline plus levodopa completed the entire study. Similarly, 26 of the 69 patients who got placebo plus levodopa finished. Many patients did not complete the study because of side effects. For those patients who did finish the study, there was a significant difference in favor of the group that got selegiline.

Overall, the selegiline plus levodopa group had lower UPDRS scores (lower scores mean fewer PD symptoms) compared to the placebo plus levodopa group. The difference in scores between the two groups actually increased as time went on. By the 4th year after adding levodopa the average difference in scores between the groups was over 6 points. By the end of the 7 years, the average UPDRS scores of the selegiline plus levodopa group were still lower than their scores from when levodopa was added. The patients who got selegiline also did not need as much levodopa to control their PD. At the 4-year point, those patients needed about 100 mg less of levodopa compared to the patients who were getting placebo. Finally, there were fewer patients on selegiline who developed the "wearing-off" fluctuation, while both groups had nearly equal percentages of dyskinesias.

Patients in the selegiline plus levodopa group had a greater tendency to have side effects from their treatment. Nausea was the most common side effect experienced.

\section{What does the study mean to patients?}

This study showed that the use of selegiline with levodopa resulted in significantly better control of PD symptoms compared to if patients were to have used levodopa alone. An especially important feature of this study is its very long duration. Because the study followed patients over many years, the investigators were able to see that patients with PD on selegiline did not worsen as much or as fast over time.

The data suggest that using selegiline in the early stages of PD, and continuing it when levodopa is needed as well, leads to less severe PD complaints. It appears that over time selegiline slows down the progression of PD symptoms. It is important to note, however, that we do not know if selegiline actually slows down the process that causes PD.

\section{Reference}

1. Pålhagen S, Heinonen E, Hågglund J, et al. Selegiline slows the progression of the symptoms of Parkinson disease. Neurology 2006; 66:1200-1206. 


\section{What is Parkinson disease?}

$\mathrm{PD}$ is a chronic, progressive movement disorder that occurs when cells in a part of the brain called the substantia nigra die. It is not known why and how the special brain cells die. These cells make a chemical called dopamine, which is used to help the brain control and coordinate the many movements our body makes. When there is reduction in the level of dopamine made in the substantia nigra, the results include symptoms of rest tremor (rhythmic shaking of a body part that only occurs when the part is not moving), rigidity or "stiffness" of body parts, bradykinesia (a term that refers to slowness of movement and trouble starting movements), and postural instability (difficulty maintaining balance and body posture, which can often lead to falls). But not every patient with PD has those four major symptoms, which sometimes makes the diagnosis of PD hard. As years go on, more cells die and the symptoms usually worsen, causing more and more problems for those patients to do their normal daily activities.

\section{What is levodopa?}

Levodopa is a medication that is converted into the brain chemical dopamine once it is ingested and transported inside specific brain cells. Therefore, it acts as a replacement for the dopamine a person with PD lacks. Although it is one of the oldest medications available for PD, it is still considered the "gold standard" medication because none of the newer medications can surpass its overall benefit on symptoms. Levodopa can cause a lot of nausea as a side effect if it is given alone; therefore, it is always given in combination with a partner medication called carbidopa, which helps to prevent nausea from happening.

\section{What are dyskinesias?}

Dyskinesias are automatic movements of a body part that look like dancing or restless motions. They have no purpose and patients have very little control over them. Dyskinesias can have different levels of severity, from being only annoying or embarrassing to being so severe that patients are not able to write, eat, or do other activities. They also can be very hard to treat. Dyskinesias tend to occur in patients who have had PD for years and have been taking levodopa for many years. Nobody knows for certain why or how dyskinesias develop, but they may be a complication of long-term levodopa use.

\section{What are "wearing-off" motor fluctuations?}

"Wearing-off" describes the problem when PD medications no longer last as long as they used to. As the disease progresses, the effect of the same amount of levodopa may last a shorter amount of time. This means that patients will feel more PD symptoms before the next time they are supposed to take a dose of medication. This can lead to anxiety, discomfort, and decreased ability to function during that period of time. "Wearing-off" is likely due to the changes that occur in the brain as PD progresses. Fortunately, there are several strategies and medications that can be used to try to treat "wearing-off."

\section{Is there a concern with using levodopa?}

Levodopa remains our best oral medication for treating PD; when compared head-to-head with other medications, it is still the most effective in treating symptoms. However, there is some thought that using levodopa for many years is associated with developing dyski- nesias. Although nobody has any definitive proof that levodopa directly causes dyskinesias, many neurologists choose to use other medications if possible before jumping straight to levodopa. This is especially true if a newly diagnosed patient with PD is relatively young (for example, 40 to 60 years old) or has mild symptoms. However, most neurologists also agree that if a patient's symptoms are so severe that the patient cannot do normal activities or is at risk for falls, then levodopa should be given in any case. It is important to always keep a patient with PD's quality of life in mind when choosing medications.

\section{What is the UPDRS?}

The UPDRS stands for Unified PD Rating Scale. It is a grading system used to measure the many symptoms of PD. It has four major sections, which measure mental function/behavior/mood, ability to do daily activities, physical performance on examination by doctors, and problems with treatment. Most of the categories are scored from 0 to 4 points, 0 being no symptom and 4 being the most severe level of the symptom. Therefore, the higher a UPDRS score, the worse a patient's PD symptoms. The UPDRS is used in most PD clinical research studies, because it allows neurologists from different groups to score and compare their patients easily.

\section{For more information \\ PD Foundation www.pdf.org}

National Parkinson's Foundation
www.parkinson.org

WE MOVE (Worldwide Education and Awareness for Movement Disorders) www.wemove.org 


\section{Neurology}

\section{Slowing down a "slow" movement disorder: Selegiline's benefit in treating Parkinson disease}

Steven E. Lo and Samay Jain

Neurology 2006;66;E28-E29

DOI 10.1212/01.wnl.0000217547.43970.ea

\section{This information is current as of April 24, 2006}

\section{Updated Information \&} Services

References

Permissions \& Licensing

Reprints including high resolution figures, can be found at: http://n.neurology.org/content/66/8/E28.full

This article cites 1 articles, 1 of which you can access for free at: http://n.neurology.org/content/66/8/E28.full\#ref-list-1

Information about reproducing this article in parts (figures,tables) or in its entirety can be found online at:

http://www.neurology.org/about/about_the_journal\#permissions

Information about ordering reprints can be found online: http://n.neurology.org/subscribers/advertise

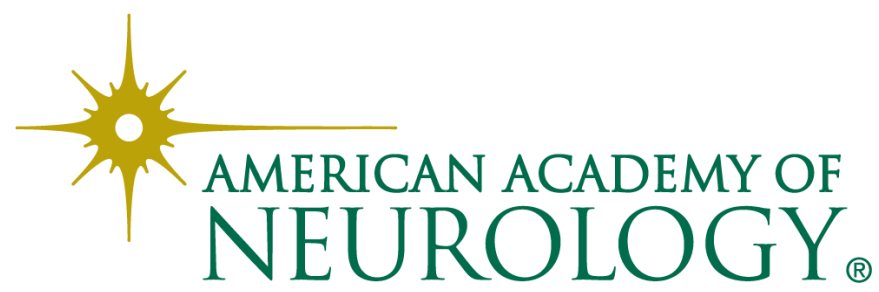

